Provided for non-commercial research and education use. Not for reproduction, distribution or commercial use.

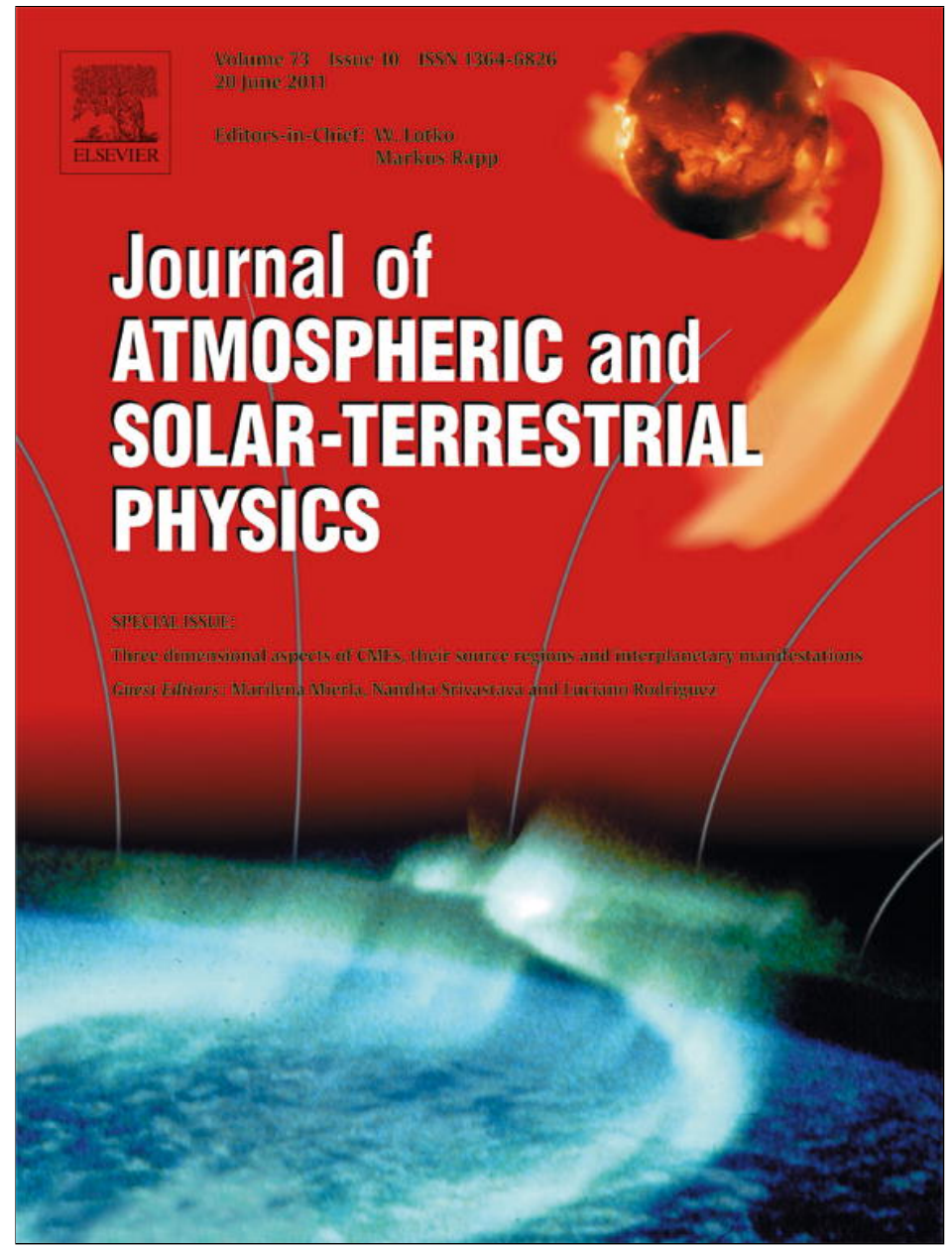

This article appeared in a journal published by Elsevier. The attached copy is furnished to the author for internal non-commercial research and education use, including for instruction at the authors institution and sharing with colleagues.

Other uses, including reproduction and distribution, or selling or licensing copies, or posting to personal, institutional or third party websites are prohibited.

In most cases authors are permitted to post their version of the article (e.g. in Word or Tex form) to their personal website or institutional repository. Authors requiring further information regarding Elsevier's archiving and manuscript policies are encouraged to visit:

http://www.elsevier.com/copyright 


\title{
Models for coronal mass ejections
}

\author{
Carla Jacobs ${ }^{\mathrm{a}, \mathrm{b}}$, Stefaan Poedts ${ }^{\mathrm{a}, \mathrm{b}, *}$ \\ a Centrum voor Plasma-Astrofysica, Celestijnenlaan 200B, 3001 Leuven, Belgium \\ ${ }^{\mathrm{b}}$ Leuven Mathematical Modeling and Computational Science Centre, Belgium
}

\section{A R T I C L E I N F O}

\section{Article history:}

Received 1 April 2010

Received in revised form 29 November 2010

Accepted 1 December 2010

Available online 15 December 2010

\section{Keywords:}

Sun

Coronal mass ejection

Solar wind

Magnetohydrodynamics

Numerical

\begin{abstract}
A B S T R A C T
Coronal mass ejections (CMEs) play a key role in space weather. The mathematical modelling of these violent solar phenomena can contribute to a better understanding of their origin and evolution and as such improve space weather predictions. We review the state-of-the-art in CME simulations, including a brief overview of current models for the background solar wind as it has been shown that the background solar wind affects the onset and initial evolution of CMEs quite substantially. We mainly focus on the attempt to retrieve the initiation and propagation of CMEs in the framework of computational magnetofluid dynamics (CMFD). Advanced numerical techniques and large computer resources are indispensable when attempting to reconstruct an event from Sun to Earth. Especially the simulations developed in dedicated event studies yield very realistic results, comparable with the observations. However, there are still a lot of free parameters in these models and ad hoc source terms are often added to the equations, mimicking the physics that is not really understood yet in detail.
\end{abstract}

(c) 2010 Elsevier Ltd. All rights reserved.

\section{Introduction}

Coronal mass ejections (CMEs) are transient solar events, occurring when bursty solar activity launches solar plasma into the heliosphere. They belong to the most violent and fascinating phenomena in the solar system. During these dramatic eruptions enormous amounts of solar material (in the range of $10^{13}-10^{16} \mathrm{~g}$ ) are ejected into the interplanetary space on a timescale of only a few hours, involving energy releases in the range $10^{20}-10^{25} \mathrm{~J}$. Typical CME velocities are in the range of $400-500 \mathrm{~km} \mathrm{~s}^{-1}$, but these can amount to $2000 \mathrm{~km} \mathrm{~s}^{-1}$ and even higher. The occurrence of CMEs varies over the solar cycle from less than 1 per day during solar minimum to more than 5 per day during solar maximum. A detailed overview of CME observations can be found for example in Yashiro et al. (2004) and Schwenn et al. (2006). CMEs used to be observed only near the Sun by means of white-light coronagraphs and their properties could also be measured near the Earth upon arrival if directed towards it. Since recently, however, they are observed in a large part of the heliosphere from outside the Sun-Earth line, viz. by the STEREO heliospheric imagers (Harrison et al., 2008). These energetic events involve large-scale changes in the coronal structure and cause significant disturbances in the solar wind. Interplanetary coronal mass ejections (ICMEs) are referred to as solar wind structures that differ from the ambient solar wind and are considered as the interplanetary counterpart of a CME (Burlaga et al., 1982). Typical ICME characteristics are, among others, significant lower solar wind

\footnotetext{
* Corresponding author at: Centrum voor Plasma-Astrofysica, Celestijnenlaan 200B, 3001 Leuven, Belgium.

E-mail address: Stefaan.Poedts@wis.kuleuven.be (S. Poedts).
}

proton temperature, the presence of counter-streaming electrons, and a smooth variation in the magnetic field (Zurbuchen and Richardson, 2006; Richardson and Cane, 2010). A subset of ICMEs show a smooth rotation of the magnetic field vector and are referred to as magnetic clouds (Démoulin, 2008). Especially the massive and fast CMEs are interesting to study as they play a key role in space weather because they usually propagate much faster than the background solar wind, giving rise to huge shock waves that propagate through the heliosphere. These shock waves can accelerate energetic particles and give rise to so-called gradual Solar Energetic Particle (SEP) events (Reames, 1999). Moreover, the interplanetary (IP) shocks, the energetic particles and the magnetic clouds caused by CMEs can interact with and disrupt the magnetosphere of the Earth, causing geomagnetic storms which can have harmful consequences for communication and navigation systems, power supplies, etc. These severe effects prove CMEs to be the most important solar drivers of space weather. Consequently, a careful study of the origin, the structure, and the propagation and evolution characteristics of these violent solar phenomena is essential for a deeper insight into space weather physics. This, in turn, is required for more accurate and reliable predictions and long-term forecasts of the space weather.

Almost four decades have past since the first CME detection (Tousey, 1973) and an abundance of CME observations became available since then. Yet, the origin nor the dynamics of these massive solar eruptions are fully understood. With more and better data available, and more studies being carried out by numerous scientists, it became clear during the last years that small scale outflow is ubiquitously observed in the corona and that solar eruptions occur on all scales (Robbrecht et al., 2009). Shearing motions in the photosphere, along the magnetic neutral line, 
rotating flows in sunspots, emergence and cancellation of new magnetic flux are all processes that are commonly observed in the pre- and post-phases of CME events, indicating a prominent role of the solar magnetic field in the process of CME initiation.

Due to the complexity of the problem, the numerical modelling of solar transients for the prediction of the space weather is a challenging task that requires a multidisciplinary approach including, e.g., advanced mathematical modelling, a large amount of computational power, the continuous improvement of numerical techniques, and the incorporation of observational data. Any physically acceptable model for solar eruptions has to be able to explain the fundamental causes of the eruption itself as well as the nature of morphological features associated with it. The ultimate goal is the modelling of solar eruptions from their birth, i.e. the onset of the eruptions, up to their interaction with the Earth (and/or other planets), and this on realistic time scales (much shorter than the time scales of the phenomena themselves if predictions are to be made).

The theoretical modelling of the evolution of CMEs can be divided into four different sub-problems: (1) The observational study and modelling of the fast and slow solar wind where questions regarding the heating source(s) and acceleration mechanism(s) of the fast wind component need to be answered. (2) The initiation of CMEs: why do CMEs occur at all, and how are they triggered? (3) The propagation and acceleration/deceleration of the CMEs and, in particular, the observed time-height curves and ICME arrival times at $1 \mathrm{AU}$ need to be explained. Also the evolution and the structure of the CMEs and their leading shock fronts during their propagation through the IP medium need to be studied. As a matter of fact, the modification(s) of the CME shock structure may contain important clues to understanding the evolution and propagation properties of CMEs. (4) The impact of the CMEs or magnetic clouds on the Earth's magnetosphere. The interaction of the CMEs with the bow shock of the Earth's magnetosphere drastically affects the characteristics of the magnetic field lines and induces complicated magnetic field line reconnections. Clearly, this affects the geo-effectiveness of the magnetic storms.

In the present paper, an overview will be given of the latest developments on the theoretical modelling of CMEs with the focus on the first three subproblems. Numerical simulations have pointed out the importance of an accurate modelling of the background medium in which the disturbances propagate. Changes in the velocity and density of the solar wind affect the dynamics and morphology of the CMEs (e.g. Wu et al., 2005; Jacobs et al., 2005). The efforts on solar wind modelling are discussed in the next section. The third section in this paper deals with the models that are currently used for CME initiation studies. Numerical CME models can be divided into two large groups: (1) models focusing on the initiation mechanism only and neglecting the presence of the solar wind and (2) models that also take into account the initial propagation of the CMEs. In the former case the simulation domain is often limited to a box with the lower boundary located in the photosphere or even in the convection zone and the upper (outer) boundary situated in the lower corona, still relatively close to the Sun. The second group of models usually include the background solar wind and have a simulation domain starting from the lower solar corona and extending to beyond the superAlfvénic region. Computer simulations investigating the propagation and dynamical evolution of CMEs are discussed in Section 4. Those models often neglect the initiation of the CME itself and focus on the propagation of the related shock wave or magnetic structure. The paper is closed by a concluding Section 5 .

\section{Modelling the background solar wind}

Since the first attempt to numerically model the solar corona by Pneuman and Kopp (1971), a lot of progress has been made. The work of Han et al. (1988) is the first which reports on a 3D, timedependent modeling of super-sonic, super-Alfvénic MHD flow from $18 R_{\odot}$ up to the Earth's environment. Nowadays, thanks to the vast increment in computational power, the usage of 3D MHD models for reconstructing the solar corona and solar wind has become even more common. Moreover, the availability of ever more detailed observations makes it possible to produce more realistic simulations, e.g. by the inclusion of the observational data through the boundary conditions. A 3D MHD simulation of the solar corona in which measurements of the line-of-sight magnetic field component served as a boundary condition for the model was first performed by Linker et al. (1999). More recently, Hayashi (2005) introduced a quasi-real time coronal MHD simulation model that employs daily-updated SOHO-MDI data as input. The real-time result, however, requires a rather coarse grid $(72 \times 32 \times 64$ for the radial, latitudinal and longitudinal direction, respectively). Hayashi (2005) assumed a polytropic plasma with a specific heat ratio of 1.05 in order to mimic the near-isothermal coronal situation due to the high thermal conduction. The polytropic assumption yields a small contrast in flow speed and density (which is less realistic), but the magnetic field quantities and the trends of these plasma variables are well retrieved. Recently, Hu et al. (2008) performed 3D MHD modeling of the global solar corona throughout solar cycle 23 , also assuming an adiabatic index $\gamma=1.05$.

A combination of an empirical and physical based representation of the quasi-steady global solar wind is the well-known WangSheeley-Arge model (Arge et al., 2004), which is an improved version of the earlier proposed model by Wang and Sheeley (1990), relating the magnetic field expansion factor to the solar wind speed. The model has been comprehensively validated with observations spanning nearly a full solar cycle (Owens et al., 2005) and is used as a boundary condition in several MHD models as well.

Roussev et al. (2003b) also constructed a three-dimensional model for the solar wind incorporating solar magnetogram observations as boundary conditions. In this model, the solar wind is powered by the energy interchange between the coronal plasma and the large-scale MHD turbulence. In order to reproduce the observed bimodal structure of the solar wind, with a fast and a slow component, these authors impose a temperature variation on the solar surface depending on the strength of the magnetic field. This solar wind model has been further developed by Cohen et al. (2007) who employ the Bernoulli integral to bridge the observed solar wind speed at $1 \mathrm{AU}$ with the assumed distribution of the polytropic index on the solar surface. Nakamizo et al. (2009) included extra source terms in both the momentum and energy equations of the MHD system to satisfactorily model the 3D solar wind during Carrington rotation (CR) 2028. These source terms decay exponentially with altitude and their strength depends on the magnetic field topology. Usmanov and Goldstein (2006) have developed a 3D steady state MHD model of the solar wind that covers the region from the coronal base up to $100 \mathrm{AU}$. This model includes the effect of Alfvén wave pressure and also accounts for the effect of pickup protons in the distant heliosphere. In their model of the solar corona, Lionello et al. (2009) included the additional energy transport terms of coronal heating, electron heat conduction, and optically thin radiative cooling into the governing MHD energy equation. They investigated the effect of several heating functions and succeeded in reproducing the EUV emission during CR 1913 fairly well. A similar study to the one of Lionello et al. (2009) was performed by Downs et al. (2010) who placed the inner boundary of their model in the upper transition region or chromosphere rather than in the low corona. They conclude that the applied heating function can have significant feedback onto the three-dimensional magnetic topology in the low corona.

Most of the MHD models can fit the measured solar wind velocity rather well, but they often have problems with the correct 
modelling of one or more of the other plasma quantities. The current state-of-the-art coronal and solar wind models differ in the physics included in the equations. Including more physics, like adding the effect of thermal conduction, radiative transport, resistive and viscous terms, complicates the numerics and are therefore often neglected. Instead, the missing physics is modelled in an ad hoc way by including additional source terms to the energy and momentum equation, where the functional form of the source term will determine the final flow profile. An accurate model for the solar wind is, however, important when studying the interplanetary evolution of a coronal mass ejection, as is known from both numerical simulations (Jacobs et al., 2005; Wu et al., 2005) and observations (Maloney and Gallagher, 2010). The background solar wind will influence the velocity, the spread angle, and the mass distribution of the CME. The aerodynamic drag force, which is determined by the solar wind speed and density, but also by the size and mass of the CME, seems to be crucial in the acceleration/deceleration of CMEs throughout their interplanetary journey (Vršnak et al., 2004, 2010)

\section{CME initiation models}

To date, to our knowledge there is no CME model sufficiently well developed to fully explain all of the observed features of solar eruptions and related phenomena. The basic pre-eruption configuration and the topological changes in the magnetic field that result in the conversion of a large fraction of the magnetic energy into kinetic energy are not yet well-known. Nevertheless, significant progress has been made in our understanding of the basic physical processes that are involved in those events. In each of the eruption models, a key role seems to be assigned to magnetic reconnection. Reviews on CME initiation mechanisms can be found in Forbes (2000, 2010), Klimchuk (2001), Low (2001), Zhang and Chye Low (2005), Moore and Sterling (2006), and Forbes et al. (2006).

Following the classification of Forbes $(2000,2010)$ and Klimchuk (2001) the current CME models can be defined as 'storage and release' models, in which the energy to drive the CME is first stored in the coronal magnetic field and later suddenly released when it crosses a threshold value. A common feature in most of the models is the presence of twisted magnetic field structures, referred to as flux ropes, either existing prior to the onset of the CME or being created during the eruption (Roussev and Sokolov, 2006). By means of numerical simulations the theoretical models can be validated and compared to the observations. Two classes of models can be considered: (1) models assuming a pre-existing coronal magnetic structure that becomes unstable and erupts and (2) models considering the rise of a magnetic flux tube from below the photosphere that expands when it reaches the corona. In what follows below, a selected overview is given of the efforts undertaken over the past decade in this field of research.

The analytical flux rope model of Lin and Forbes (2000) is a 2D model of a flux rope in equilibrium. Two surface point sources anchor the flux rope in a magnetic arcade. By moving the point sources together at a rate much slower than the Alfvén time scale, a loss of equilibrium happens and the flux rope moves rapidly upward. Numerical simulations of a similar model were carried out by Chen and Shibata (2000) who proposed an emerging magnetic flux triggering mechanism for the onset of CMEs. These authors have performed two dimensional MHD simulations in a Cartesian box of a flux rope that is initially in equilibrium and that is then made unstable by the emergence of extra magnetic flux trough the lower boundary. A current sheet then forms below the flux rope and the flux rope is pushed upward by fast magnetic reconnection. Dubey et al. (2006) have extended this study in spherical geometry. A 3D analytical model for a flux rope in equilibrium was developed by Titov and Démoulin (1999), which had been proposed to explain flares and CMEs. Roussev et al. (2003a) incorporated the Titov and Démoulin (1999) model in a 3D MHD simulation. It turned out that a high amount of twist was necessary in order to manyfest any loss of equilibrium. However, the flux rope never evolved into an erupting CME. They concluded that the strength of the overlying field falls off too slowly in Titov and Démoulin (1999) model in order to permit a CME. Also Török et al. (2004) and Török and Kliem (2005) investigated the stability of Titov and Démoulin (1999) model using zero- $\beta$ MHD simulations. They concluded that the model is kink unstable when a critical amount of twist is exceeded. By modifying the model such that the overlying field drops more rapidly with height above the flux rope, they obtained a full eruption of the system.

Another subset of CME initiation models relies on the existence of coronal magnetic arcades, which are sheared and become unstable and erupt once some critical state is reached in the solar corona. In contrast to the previous subset of models, here a magnetic flux rope does initially not exists, but is formed by magnetic reconnection in the course of the dynamic evolution of the system. Mikic and Linker (1994) had shown already by means of 2.5D MHD simulations that shearing motions energize the coronal magnetic field and might cause the formation of a flux rope provided the shearing motions continue for a sufficiently long time. A parameter study of the effect of the shear velocity and the background wind on the evolution of the flux rope formation when driven by shearing motions was performed by Jacobs et al. (2006). In case the coronal magnetic field has a multipolar topology, creating a triple arcade system, where the polarity of the inner arcade is opposite to that of the overlying arcade, the conditions are suitable for the 'breakout' mechanism (Antiochos et al., 1999). Shearing motions applied at the inner boundary cause the central arcade to expand upward and reconnect with the overlying field. Magnetic flux is transferred from the central arcade system to the side arcades. The removal of the overlying magnetic field facilitates the outward motion of the central arcade. Finally, reconnection below the central arcade will set in and cause the formation of a flux rope. The 3D counterpart of the breakout mechanism was recently simulated by DeVore and Antiochos (2008) and Lynch et al. (2008, 2009). The previously mentioned simulations of the breakout model ignore the effect of the solar wind. van der Holst et al. $(2007,2009)$ demonstrated that including the background solar wind affects the evolution of the system. The solar wind shapes the overlying magnetic field into a helmet streamer structure. At first instance the evolution of the system is similar to that of the classic breakout model. However, from a certain moment reconnection sets in on the sides of the erupting central arcade and the breakout reconnection stops, eventually resulting in the detachment of the helmet streamer. Within the breakout model, Zuccarello et al. (2009) investigated, by means of 2.5D MHD simulations, both emergence of (radial) magnetic flux and shearing motions along the magnetic inversion line as possible driver mechanisms for CMEs. By applying time-dependent boundary conditions at the inner (lower) boundary, the central arcade of the multi-flux system first expands, leading to the eventual eruption of the top of the helmet streamer, as in van der Holst et al. (2007). The topological and dynamical evolution of the system (when driven by the two different types of boundary conditions) have been compared. It has been shown that, in this $2.5 \mathrm{D}$ setup at least, both driving mechanisms result in a slow CME and that the overall evolution of the system is independent of the driving mechanism: the actual CME is the detached helmet streamer. However, the evolution of the central arcade is different in the two cases. The central arcade eventually becomes a flux rope in the shearing case whereas in the flux emergence case there is no formation of a flux rope. These simulations showed that magnetic helicity is not crucial for occurrence of a solar eruption. However, if helicity is present, a specific threshold value exists. See Figs. 1 and 2 for an illustration of this simulation. Jacobs et al. (2009) 
a

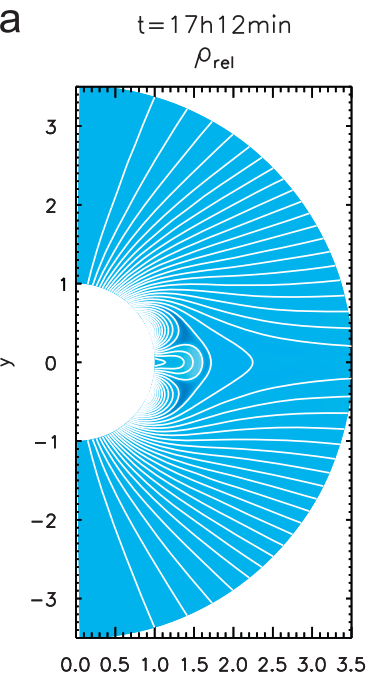

b

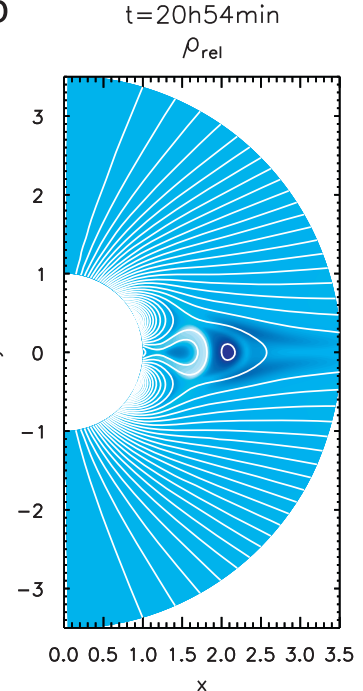

C

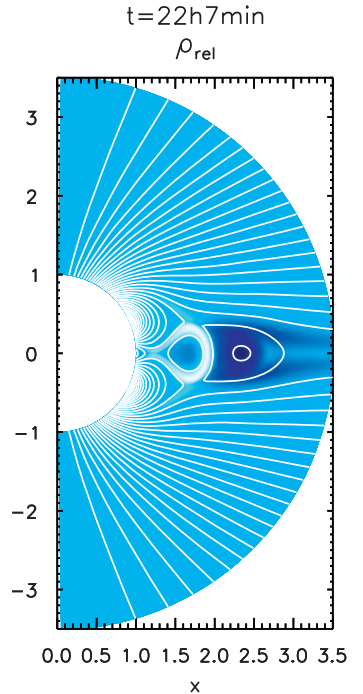

d

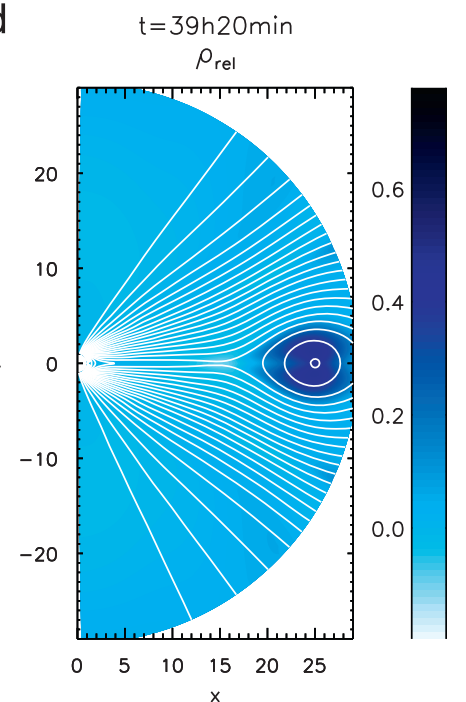

Fig. 1. By means of shearing motions, the central arcade expands and rises. Shown are the contours of the relative density (colour coded) and some magnetic field lines (white lines). Panel (a) shows the expanding central arcade ( $17 \mathrm{~h} 12 \mathrm{~min}$ ). Panel (b) shows the increase of the density inside the helmet streamer and the further expansion of the central arcade ( $20 \mathrm{~h} 54 \mathrm{~min}$ ). Panel (c) shows the presence of the two flux ropes ( $22 \mathrm{~h} 7 \mathrm{~min}$ ), and panel (d) shows the actual CME leaving the computational domain ( $39 \mathrm{~h}$ $20 \mathrm{~min}$ ). After Zuccarello et al. (2009).
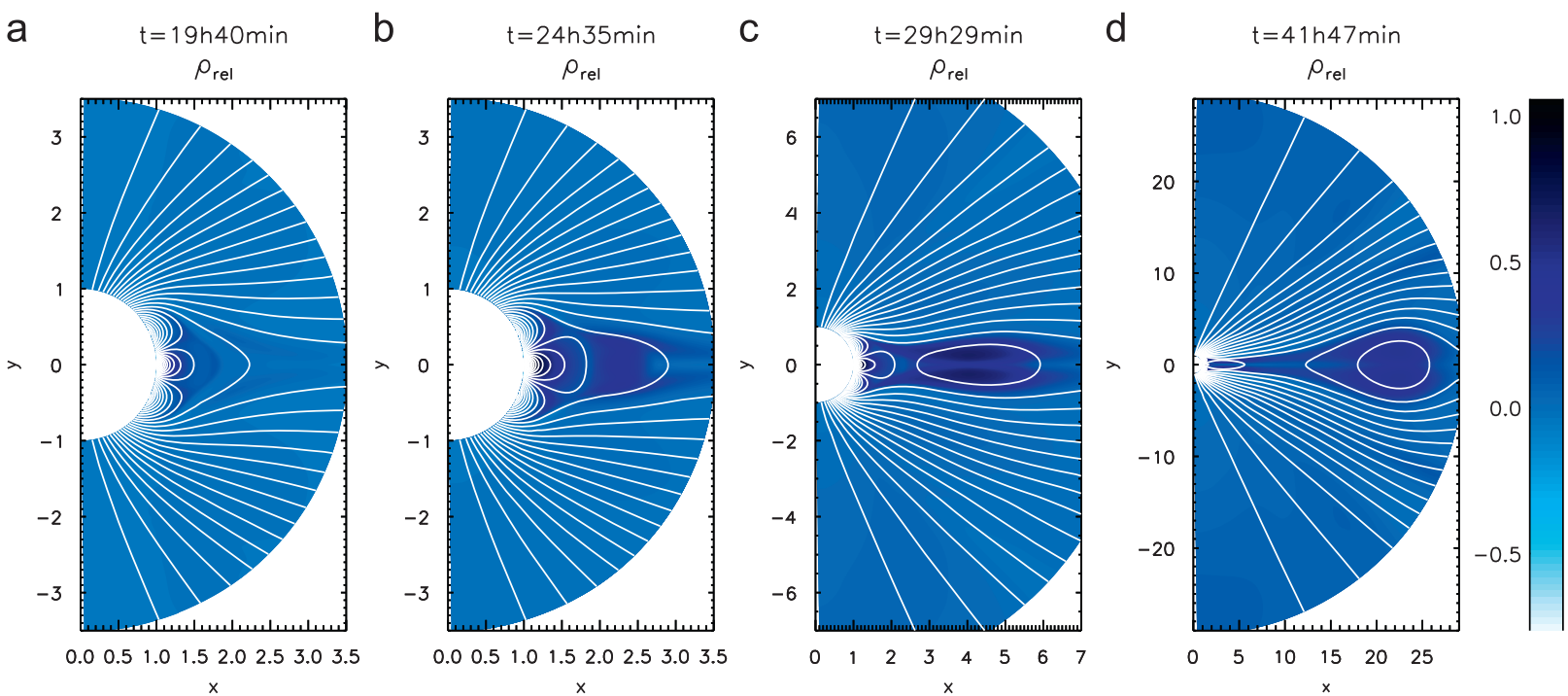

Fig. 2. By means of magnetic flux emergence, the central arcade expands and rises. Shown are the contours of the relative density (colour coded) and some magnetic field lines (white lines). Panel (a) shows the expanding central arcade (19 h $40 \mathrm{~min}$ ). Panel (b) shows the increase of the density inside the helmet streamer and the further expansion of the central arcade when all the flux has emerged ( $24 \mathrm{~h} 35 \mathrm{~min}$ ). The panels (c) and (d) show the actual CME propagating ( $29 \mathrm{~h} 29 \mathrm{~min}$ ) and leaving the computational domain (41 h 47 min). After Zuccarello et al. (2009).

investigated the internal structure of a $3 \mathrm{D}$ coronal mass ejection (CME) and its dynamics by invoking an initiation mechanism in a quadrupolar magnetic setting superimposed on an idealized model of the solar corona. A fast CME is obtained by applying shearing motions resembling flux emergence at the solar boundary (similar to Roussev et al., 2007), energizing the initial equilibrium field until it eventually erupts. The simulated CME shows the typical characteristics of a magnetic cloud (MC) as it propagates away from the Sun and interacts with the bimodal solar wind. However, no distinct flux rope structure is found to be present in the associated interplanetary ejection. The observed rotation of the magnetic field vector, characteristic of a MC, results from the creation of significant writhe in the CME's magnetic field by a series of magnetic reconnection events between the eruptive magnetic field and the ambient field. Modifying the polarity of the overlying field, changes the initial configuration to the classical breakout setup with only one coronal null point. Applying the same driving mechanism then results in a slower CME (see Figs. 3-5).

In the magnetic flux-cancellation models a flux rope is formed by reconnecting the opposite polarity feet of a sheared magnetic arcade (e.g. Amari et al., 2003; Linker et al., 2003; Roussev et al., 2004; Riley et al., 2006; Reeves et al., 2010). Amari et al. (2008) applied flux cancellation on both a dipolar and quadrupolar (breakout) setting. These authors found a similar behaviour for both configurations: (1) during the first phase, the coronal magnetic field evolves slowly, with a twisted flux rope in equilibrium being created at some time and (2) non-equilibrium sets in at a critical time and the configuration experiences a major global disruption. The existence of null points in the magnetic configuration influences the dynamics and energetics of the eruption. Mackay and Van Ballegooijen (2006) investigated the evolution 

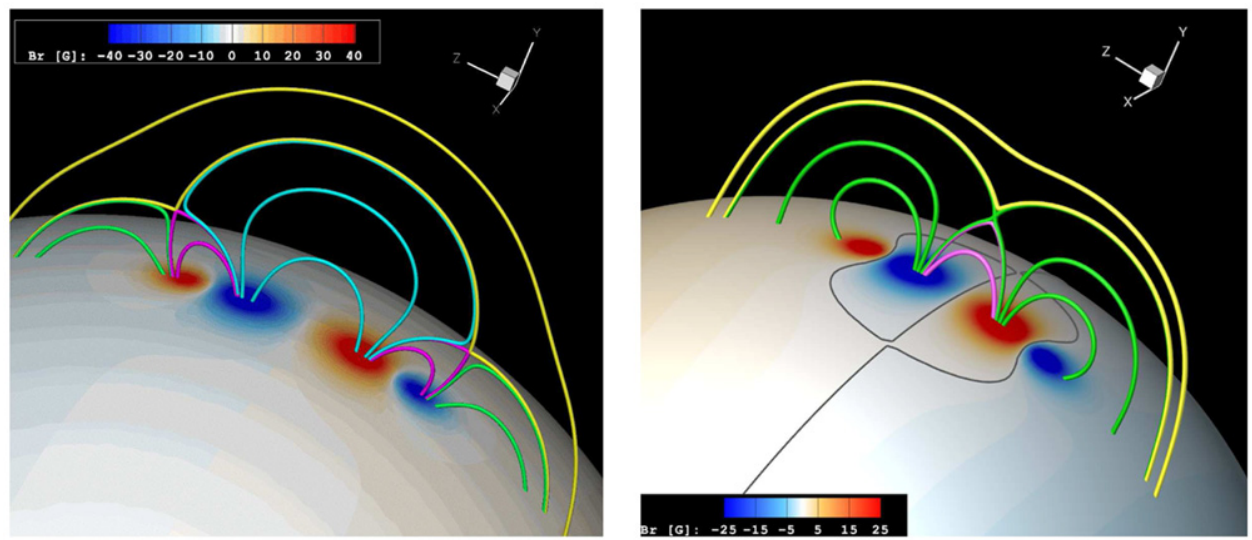

Fig. 3. The initial magnetic configuration. Depending on the orientation of the ambient coronal magnetic field, a different topology of the magnetic field exist. The state on the left is referred to as the 'quadrupolar' case, and the state on the right as the 'dipolar' case, since the polarity of the outer magnetic spots is the same as that of the global dipole. After Jacobs et al. (2009).

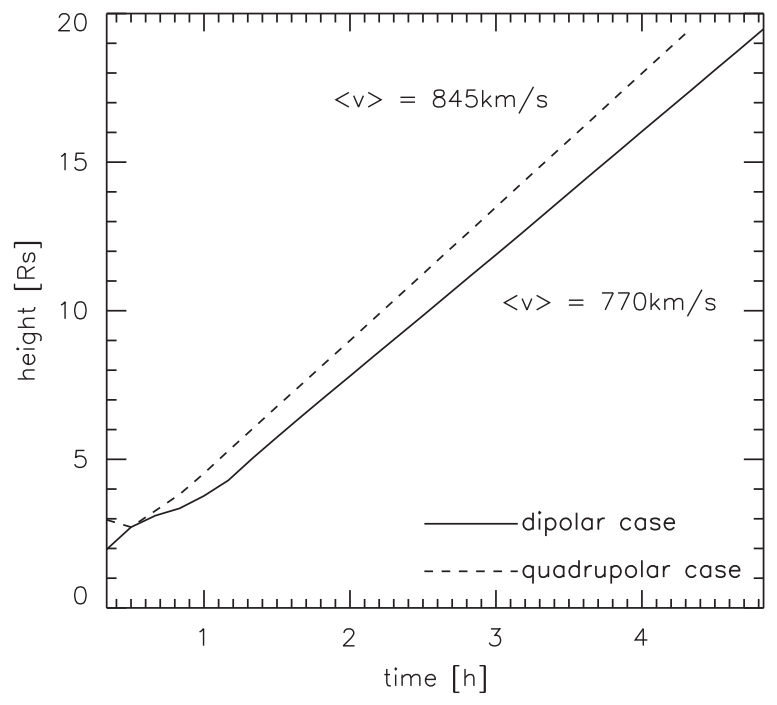

Fig. 4. Boundary driven motions enable the eruption of a CME Jacobs et al. (2009) Applying the same boundary motions on the initial states presented in Fig. 3 results in a different dynamical behavior.

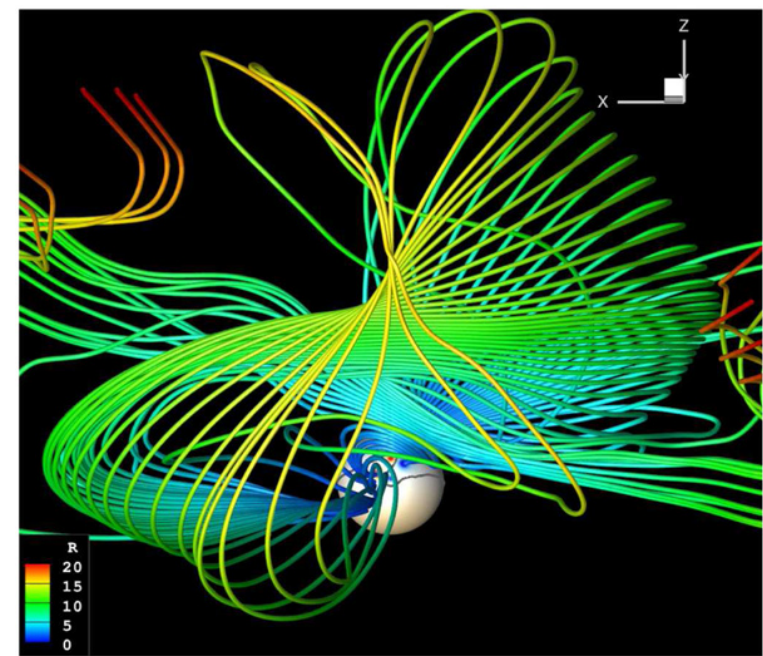

Fig. 5. Snapshot of the simulation with the 'quadrupolar' initial configuration. The magnetic field shows a strong writhe due to reconnection with the ambient solar magnetic field. After Jacobs et al. (2009). of two twisted dipoles when subducted to solar differential rotation, small scale convective flows, and magnetic diffusion. The magnetic diffusion will cause flux cancellation between the two dipoles, while the solar rotation will modify the orientation of the neutral lines. Flux ropes form between the two dipoles, and also inside the dipoles. Reconnection at the quasi separatrix layer beneath the flux ropes will cause a lift-off and eruption of the flux rope. Yeates and Mackay (2009) applied this technique in a full Sun simulation, driven by observational input of emerging active regions over a 177 day period in 1999 and they were able to reproduce $50 \%$ of the observed CME rate. In a recent work, Aulanier et al. (2010) analysed in detail the physical mechanisms that form a 3D coronal flux rope and the cause of its eruption within zero- $\beta$ MHD. By applying vortex flows and magnetic diffusion, the latter introducing flux cancellation, an asymmetric bipolar region is made unstable. Due to tether cutting reconnection a flux rope is formed that undergoes a slow rise, but it is the torus instability that finally causes the eruption.

The last class of simulations discussed are those considering the evolution of a twisted magnetic flux rope from below the photosphere into the corona, representing emergence of magnetic flux. Manchester et al. (2004b) followed the evolution of a buoyantly driven flux rope, passing from below the photosphere into the corona. Their simulation showed the self-consistent development of shear flows during the emergence of the flux system as a result of the force imbalance between the Lorentz force and the gravitational stratification of the surrounding atmosphere. The shear flows play an essential role in the separation of the upper part of the erupted flux rope, which eventually erupts. The recent simulation of Fang et al. (2010) included radiative loss, a non-ideal equation of state, and empirical coronal heating, attempting to reproduce the buoyant rise of a twisted flux tube from a granular convection zone into the low corona. The convection, absent in the simulation of Manchester et al. (2004b), has an important impact on the morphology and evolution of the erupting structure. Velocity shear and magnetic field shear are self-consistently introduced near the polarity inversion line, with a magnitude matching the observations. Archontis and Török (2008) also performed a simulation of a sub-photospheric twisted flux tube that rises from the solar interior and expands into the corona. A flux rope is formed within the expanding field, due to shearing and reconnection of field lines at low atmospheric heights. If the tube emerges into a nonmagnetized atmosphere, the flux rope rises, but remains confined inside the expanding magnetized volume. In contrast, if the expanding tube is allowed to reconnect with a pre-existing coronal field, the flux rope experiences a full eruption with a rise profile that 
is in qualitative agreement with erupting filaments and CMEs. This results points out once more the importance of the overlying magnetic field in the onset of CMEs. Also Fan and Gibson (2007) concluded that the overlying magnetic field is important for the evolution of CMEs. In their simulation of a flux rope emerging through the photosphere, these authors varied the magnetic field strength of the overlying arcades. In the case with the strongest overlying field, the emerging flux rope remained confined at first and showed kinks later on, leading to an eruption. When the overlying magnetic field declines more rapidly with height, the emerging flux rope is found to lose equilibrium and erupt via the torus instability. This confirms the previous results of Török et al. (2004), Török and Kliem (2005), and Kliem and Török (2006). It is interesting to note that in both cases studied by Fan and Gibson (2007) the normalized relative magnetic helicity of the entire coronal magnetic field is of similar magnitude at the moment when the eruption takes place $(\approx-0.18)$. This value is similar to the found by Zuccarello et al. (2009).

\section{CME propagation and evolution}

The propagation of CMEs through the inner heliosphere are often modelled ignoring the actual initiation of the CME. Placing the inner boundary of the simulation domain beyond the critical point where the solar wind becomes super fast (e.g. at $r>18 R_{\odot}$ ) has considerable advantages from a numerical point of view, since all characteristic waves are propagating in the domain, and as such all the variables can be specified at the boundary (e.g. Han et al., 1988; Vandas et al., 2002; Odstrcil et al., 2005; Kataoka et al., 2009). However, there are little or no observable parameters at these large coronal distances to constrain the boundary conditions. Wu et al. (2007b,a) developed an advanced hybrid code, called HAFv.2+3DMHD, that combines the HakamadaAkasofu-Fry simulation code version 2 (HAFv.2 Fry et al., 2003) and a fully three-dimensional, time-dependent MHD simulation code. This hybrid code was then used to study the global ICME from the 12 May 1997 solar event and the multiple eruptions during the 25-28 October 2003 solar activity. The solar wind structure was first simulated from the photosphere out to $2.5 R_{\odot}$, based on daily solar magnetogram data. The HAFv. 2 code was then used from $2.5 R_{\odot}$ to provide input at $18 R_{\odot}$ $(0.08 \mathrm{AU})$ for the three-dimensional MHD code that calculated the evolution of solar wind plasma and interplanetary magnetic field beyond this distance into the heliosphere.

An example of a 3D CME propagation model is the theoretical model of Gibson and Low (1998). This analytical model was used as a CME generation mechanism in numerical simulations (e.g. Manchester et al., 2004a; Lugaz et al., 2005), in which the dynamics of the CME are followed as it interacts with a bimodal background solar wind. An event study of three interacting CMEs was performed by Lugaz et al. (2007). As model for the background solar wind the varying polytropic index model of Roussev et al. (2003b) was used and the CMEs are initiated using an out-of-equilibrium semicylindrical flux rope (Roussev et al., 2003a). These kind of simulations provide basic physical insight into how an ICME propagates and interacts with the large-scale solar wind or with a preceding disturbance and the results of these studies can be compared with simple kinematic models (Riley and Crooker, 2004; Vršnak et al., 2010).

Numerical simulations are necessary and complementary to the observations. Especially since the STEREO era began, i.e. when the tracking of CMEs through the heliosphere became possible, numerical simulations turn out to be indispensable for a correct interpretation of the complex features seen in the data. For example, Lugaz et al. (2008, 2009) simulated in a 3D MHD model the 2007 January 24-25 solar eruptions, which were tracked by the heliospheric imagers (HI) on board of STEREO. The line-of-sight images obtained from the simulations where in excellent agreement with the real line-of-sight images. These kind of simulations allow the study of the 3D nature of CMEs and discern between observational effects (i.e. projection effect, instrumental noise, etc.) and physical effects.

\section{Conclusions}

In the last couple of years, the modelling of the solar wind and of CMEs superposed on this wind has advanced to a stage where individual events can be simulated rather realistically so that a detailed comparison with observational data is worthwhile. The current state-of-the-art 3D MHD models apply magnetogram data as input boundary conditions and even include non-MHD effects, such as solar particle acceleration and kinetic effects. Still, very few of them are sufficiently developed to explain the real events in detail, and most of them only consider one of the sub-problems (discussed above) involved in space weather modelling. Future models should aim to model the entire process from CME initiation to CME evolution selfconsistently. However, this is a difficult task as there are considerable variations of the physical conditions in the solar photosphere, corona, and IP space that involve many physical processes occurring on vastly different spatial and temporal scales. One way to deal with this problem, is to decouple the solar corona model from a model solving the inner heliosphere and to use the output of the coronal model as a boundary condition for the heliospheric model (Odstrcil et al., 2004). Coronal models need to simulate more complex physical processes, while heliospheric models can use simpler approximations over a much larger spatial domain. Computationally, it is thus more efficient to advance the heliospheric portion of the simulation independently of the coronal time step.

Another way to deal with the varying spatial scales is the use of adaptive mesh refinement techniques, as applied e.g. in the BATS-R-US code (Powell et al., 1999), or in the AMR-VAC code (van der Holst and Keppens, 2007). Currently, only two groups have developed advanced 3D coupled MHD models to model a CME event from its initiation up to the interaction with the magnetosphere of the Earth: the CORHEL model (Luhmann et al., 2004) at the University of Boston and the Space Weather Modeling Framework (SWMF) (Tóth et al., 2005) at the University of Michigan. In the SWMF, one has the choice between different models for the solar background wind as well as for different CME triggering models. Using reasonably high spatial resolutions in all of the coupled components, the SWMF runs significantly faster than real time, at least on massive parallel supercomputers. The Community Coordinated Modelling Center (CCMC, http://ccmc.gsfc.nasa.gov/models/index.php) attempts to collect the available models for space science research, covering the domain from the solar corona to the Earth's atmosphere, and make them accessible for the wide research community.

The continuous development of new numerical schemes, the ever improving CPU power and computer memory, and the ever more detailed observations, will undoubtedly lead to more realistic models of solar transients. Hence, these models will provide us with more insight into the physics involved and their predictive capabilities will increase.

\section{Acknowledgements}

This research was funded by Projects GOA/2009-009 (K.U.-Leuven), G.0304.07 (FWO-Vlaanderen), 3E090665 (FWO-Vlaanderen), and C 90347 (ESA Prodex 9). Financial support by the European Commission through the SOLAIRE Network (MTRN-CT-2006-035484) and support from the European Commission's Seventh Framework Programme (FP7/2007-2013) under the Grant nr. 218816 (SOTERIA project) is gratefully acknowledged. 


\section{References}

Amari, T., Aly, J., Mikic, Z., Linker, J., 2008. Coronal mass ejection initiation and complex topology configurations in the flux cancellation and breakout models. Astrophys. J. 671, L189-L192.

Amari, T., Luciani, J.F., Aly, J.J., Mikic, Z., Linker, J., 2003. Coronal mass ejection: initiation, magnetic helicity, and flux ropes. II. Turbulent diffusion-driven evolution. Astrophys. J. 595, 1231-1250.

Antiochos, S., DeVore, C., Klimchuk, J., 1999. A model for solar coronal mass ejections. Astrophys. J. 510, 485-493.

Archontis, V., Török, T., 2008. Eruption of magnetic flux ropes during flux emergence. Astron. Astrophys. 492, L35-L38.

Arge, C., Luhmann, J.G., Odstrcil, D., Schrijver, C.J., Li, Y., 2004. Stream structure and coronal sources of the solar wind during the May 12th, 1997 CME. J. Atmos. Sol. Terres. Phys. 66, 1295-1309.

Aulanier, G., Török, T., Démoulin, P., DeLuca, D.D., 2010. Formation of torus-unstable flux ropes and electric currents in erupting sigmoids. Astrophys. J. 708, 314-333.

Burlaga, L.F., Klein, L., Sheeley Jr., N.R., Michels, D.J., Howard, R.A., Koomen, M.J., Schwenn, R., Rosenbauer, H., 1982. A magnetic cloud and a coronal mass ejection. Geophys. Res. Lett. 9, 1317-1320.

Chen, P., Shibata, K., 2000. An emerging flux trigger mechanism for coronal mass ejections. Astrophys. J. 545, 524-531.

Cohen, O., Sokolov, I.V., Roussev, I.I., Arge, C.N., Manchester, W.B., Gombosi, T.I., Frazin, R.A., Park, H., Butala, M.D., Kamalabadi, F., Velli, M., 2007. A semiempirical magnetohydrodynamical model of the solar wind. Astrophys. J. 654, L163-L166.

Démoulin, P., 2008. A review of the quantitative links between CMEs and magnetic clouds. Ann. Geophys. 26, 3113-3125.

DeVore, C., Antiochos, S., 2008. Homologous confined filament eruptions via magnetic breakout. Astrophys. J. 680, 740-756.

Downs, C., Roussev, I.I., van der Holst, B., Lugaz, N., Sokolov, I.V., Gombosi, T.I., 2010 Toward a realistic thermodynamic magnetohydrodynamic model of the global solar corona. Astrophys. J. 712, 1219-1231.

Dubey, G., van der Holst, B., Poedts, S., 2006. The initiation of coronal mass ejections by magnetic flux emergence. Astron. Astrophys. 459, 927-934.

Fan, Y., Gibson, S., 2007. Onset of coronal mass ejections due to loss of confinement of coronal flux ropes. Astrophys. J. 668, 1232-1245.

Fang, F., Manchester, W., Abbett, W.P., van der Holst, B., 2010. Simulation of flux emergence from the convection zone to the corona. Astrophys. J. 714, $1649-1657$

Forbes, T., 2010. Models of coronal mass ejections and flares. In: Schrijver, C.J., Siscoe, G.L. (Eds.), Heliophysics-Space Storms and Radiation: Causes and Effects. Cambridge University Press, pp. 159-191 (Chapter 6).

Forbes, T.G., 2000. A review on the genesis of coronal mass ejections. J. Geophys. Res. $105,23153-23165$.

Forbes, T.G., Linker, J.A., Chen, J., Cid, C., Kóta, J., Lee, M.A., Mann, G., Mikić, Z., Potgieter, M.S., Schmidt, J.M., Siscoe, G.L., Vainio, R., Antiochos, S.K., Riley, P. 2006. CME Theory and Models. Space. Sci. Rev. 123, 251-302.

Fry, C.D., Dryer, M., Smith, Z., Sun, W., Deehr, C.S., Akasofu, S., 2003. Forecasting solar wind structures and shock arrival times using an ensemble of models. J. Geophys. Res. 108 1070-+

Gibson, S.E., Low, B.C., 1998. A time-dependent three-dimensional magnetohydrodynamic model of the coronal mass ejection. Astrophys. J. 493, 460-473.

Han, S.M., Wu, S.T., Dryer, M., 1988. A three-dimensional, time-dependent numerical modeling of super-sonic super-alfvnic MHD flow. Comput. Fluids 16, 81-103.

Harrison, R., Davis, C., Eyles, C., Bewsher, D., Crothers, S., Davies, J., Howard, R., Moses, D., Socker, D., Newmark, J., Halain, J.P., Defise, J.M., Mazy, E., Rochus, P., Webb, D. Simnett, G., 2008. First imaging of coronal mass ejections in the heliosphere viewed from outside the Sun-Earth line. Sol. Phys. 247, 171-193.

Hayashi, K., 2005. Magnetohydrodynamic simulations of the solar corona and solar wind using a boundary treatment to limit solar wind mass flux. Astrophys. J. Ser. $161,480-494$.

Hu, Y.Q., Feng, X.S., Wu, S.T., Song, W.B., 2008. Three-dimensional MHD modeling of the global corona throughout solar cycle 23. J. Geophys. Res. Space Phys. 113 3106-+.

Jacobs, C., Poedts, S., van der Holst, B., 2006. The effect of the solar wind on CME triggering by magnetic foot point shearing. Astron. Astrophys. 450, 793-803.

Jacobs, C., Poedts, S., van der Holst, B., Chané, E., 2005. On the effect of the background wind on the evolution of interplanetary shocks. Astron. Astrophys. 430, 1099-1107.

Jacobs, C., Roussev, I.I., Lugaz, N., Poedts, S., 2009. The internal structure of corona mass ejections: are all regular magnetic clouds flux ropes? Astrophys. J. 695 L171-L175.

Kataoka, R., Ebisuzaki, T., Kusano, K., Shiota, D., Inoue, S., Yamamoto, T., Tokumaru, M. 2009. Three-dimensional MHD modeling of the solar wind structures associated with 13 December 2006 coronal mass ejection. J. Geophys. Res. 114, A10102.

Kliem, B., Török, T., 2006. Torus instability. Phys. Rev. Lett. 96, 255002.

Klimchuk, J.A., 2001. Theory of coronal mass ejections. In: Song, P., Singer, H.J. Siscoe, G.L. (Eds.), Space Weather. Geophysical Monograph Series, vol. 125. AGU, p. 143.

Lin, J., Forbes, T.G., 2000. Effects of reconnection on the coronal mass ejection process. J. Geophys. Res. 105, 2375-2392.

Linker, J., Mikić, Z., Biesecker, A., Forsyth, R.J., Gibson, S.E., Lazarus, A.J., Lecinski, A. Riley, P., Szabo, A., Thompson, B.J., 1999. Magnetohydrodynamic modeling of the solar corona during Whole Sun Month. J. Geophys. Res. 104, 9809-9830.
Linker, J.A., Mikić, Z., Lionello, R., Riley, P., Amari, T., Odstrcil, D., 2003. Flux cancellation and coronal mass ejections. Phys. Plas. 10, 1971-1978.

Lionello, R., Linker, J.A. Mikić, Z., 2009. Multispectral emission of the Sun during the first whole Sun month: magnetohydrodynamic simulations. Astrophys. J. 690 902-912.

Low, B.C., 2001. Coronal mass ejections, magnetic flux ropes, and solar magnetism. J. Geophys. Res. 106, 25141-25163.

Lugaz, N., Manchester, W.B., Gombosi, T.I., 2005. The evolution of coronal mass ejection density structures. Astrophys. J. 627, 1019-1030.

Lugaz, N., Manchester, W.B., Roussev, I.I., Tóth, G., Gombosi, T.I., 2007. Numerical investigation of the homologous coronal mass ejection events from the active region 9236. Astrophys. J. 659, 788-800.

Lugaz, N., Vourlidas, A., Roussev, I.I., Jacobs, C., Manchester, IV.W.B., Cohen, O., 2008 The brightness of density structures at large solar elongation angles: what is being observed by STEREO SECCHI? Astrophys. J. 684, L111-L114.

Lugaz, N., Vourlidas, A., Roussev, I.I., Morgan, H., 2009. Solar terrestrial simulation in the STEREO Era: the 24-25 January 2007 eruptions. Sol. Phys. 256, 269-284.

Luhmann, J.G., Solomon, S.C., Linker, J.A., Lyon, J.G., Mikić, Z., Odstrcil, D., Wang, W., Wiltberger, M., 2004. Coupled model simulation of a Sun-to-Earth space weather event. J. Atmos. Sol. Terres. Phys. 66, 1243-1256.

Lynch, B., Antiochos, S., DeVore, C., Luhmann, J., Zurbuchen, T., 2008. Topological evolution of a fast magnetic breakout CME in three dimensions. Astrophys. J. 683, 1192-1206.

Lynch, B., Antiochos, S.K., Li, Y., Luhmann, J.G., DeVore, C.R., 2009. Rotation of coronal mass ejections during eruption. Astrophys. J. 697, 1918-1927.

Mackay, D., Van Ballegooijen, A.A., 2006. Models for the large scale corona. I. Formation, evolution, and liftoff of magnetic flux ropes. Astrophys. J. 641, 577-589.

Maloney, S.A., Gallagher, P.T., 2010. Solar wind drag and the kinematics of interplanetary coronal mass ejections. Astrophys. J. 724, L127-L132.

Manchester, W., Gombosi, T., Roussev, I., Ridey, A., De Zeeuw, D., Sokolov, I., Powell, K., Tóth, G., 2004a. Modeling a space weather event from Sun to the Earth: CME generation and interplanetary evolution. J. Geophys. Res. 109, A02107.

Manchester, W.B., Gombosi, T.I., DeZeeuw, D.L., Fan, Y., 2004b. Eruption of a buoyantly emerging magnetic flux rope. Astrophys. J. 610, 588-596.

Mikić, Z., Linker, J., 1994. Disruption of coronal magnetic field arcades. Astrophys. J. $430,898-912$.

Moore, R.L., Sterling, A.C., 2006. Initiation of coronal mass ejections. In: Gopalswamy, N., Mewaldt, R., Torsti, J. (Eds.), Solar Eruptions and Energetic Particles. Monograph Series, vol. 165. AGU, p. 43.

Nakamizo, A., Tanaka, T., Kubo, Y., Kamei, S., Shimazu, H., Shinagawa, H., 2009 Development of the 3-D MHD model of the solar corona-solar wind combining system. J. Geophys. Res. 114 7109-+.

Odstrcil, D., Pizzo, V.J., Arge, C.N., 2005. Propagation of the 12 May 1997 interplanetary coronal mass ejection in evolving solar wind structures. J. Geophys. Res. 110, A02106.

Odstrcil, D., Pizzo, V.J., Linker, J.A., Riley, P., Lionello, R., Mikić, Z., 2004. Initial coupling of coronal and heliospheric numerical magnetohydrodynamics codes. J. Atmos. Sol. Terres. Phys. 66, 1311-1320.

Owens, M.J., Arge, C.N., Spence, H.E., Premboke, A., 2005. An event-based approach to validating solar wind speed predictions: high-speed enhancements in the Wang-Sheeley-Arge model. J. Geophys. Res. 110, A12105.

Pneuman, G.W., Kopp, R.A., 1971. Gas-magnetic field interactions in the solar corona. Sol. Phys. 18, 258-270.

Powell, K.G., Roe, P.L., Linde, T.J., Gombosi, T.I., DeZeeuw, D.L., 1999. A solutionadaptive upwind scheme for ideal magnetohydrodynamics. J. Comput. Phys. $154,284-309$.

Reames, D.V., 1999. Particle acceleration at the Sun and in the heliosphere. Space. Sci. Rev. 90, 413-491.

Reeves, K.K., Linker, J.A., Mikić, Z., Forbes, T.G., 2010. Current sheet energetics, flare emissions, and energy partition in a simulated solar eruption. Astrophys. J. 721 1547-1558.

Richardson, I.G., Cane, H.V., 2010. Near-earth interplanetary coronal mass ejections during solar cycle 23 (1996-2009): catalog and summary of properties. Sol. Phys. 264, 189-237.

Riley, P., Crooker, N.U., 2004. Kinematic treatment of coronal mass ejection evolution in the solar wind. Astrophys. J. 600, 1035-1042.

Riley, P., Linker, J.A., Mikić, Z., Odstrcil, D., 2006. Modeling interplanetary corona mass ejections. Adv. Space. Res. 38, 535-546.

Robbrecht, E., Berghmans, D., Van der Linden, R.A.M., 2009. Automated LASCO CME catalog for solar cycle 23: are CMEs Scale invariant? Astrophys. J. 691, $1222-1234$.

Roussev, I.I., Forbes, T.G., Gombosi, T.I., Sokolov, I.V., DeZeeuw, D.L., Birn, J., 2003a. A three-dimensional flux rope model for coronal mass ejections based on a loss of equilibrium. Astrophys. J. 588, L45-L48.

Roussev, I.I., Gombosi, T.I., Sokolov, I.V., Velli, M., Manchester, W.B., DeZeeuw, D.L., Liewer, P., Tóth, G., Luhmann, J., 2003b. A three-dimensional model for the solar wind incorporating solar magnetogram observations. Astrophys. J. 595, L57-L60.

Roussev, I.I., Lugaz, N., Sokolov, I.V., 2007. New physical insight on the changes in the magnetic field topology during coronal mass ejections: case studies for the 2002 April 21 and 2002 August 24 events. Astrophys. J. 668, L87-L90.

Roussev, I.I., Sokolov, I.V., 2006. Models of solar eruptions: recent advances from theory and simulations. In: Gopalswamy, N., Mewaldt, R., Torsti, J. (Eds.), Solar Eruptions and Energetic Particles. Monograph Series, vol. 165. AGU, pp. 89-102. 
Roussev, I.I., Sokolov, I.V., Forbes, T.G., Gombosi, T.I., Lee, M.A., Sakai, J.I., 2004. A numerical model of a coronal mass ejection: shock development with implications for the acceleration of GeV protons. Astrophys. J. 605, L73-L76.

Schwenn, R., Raymond, J.C., Alexander, D., Ciaravella, A., Gopalswamy, N., Howard, R., Hudson, H., Kaufmann, P., Klassen, A., Maia, D., Munoz-Martinez, G., Pick, M., Reiner, M., Srivastava, N., Tripathi, D., Vourlidas, A., Wang, Y., Zhang, J., 2006. Coronal observations of CMEs. Report of Working Group A. Space. Sci. Rev. 123, 127-176.

Titov, V.S., Démoulin, P., 1999. Basic topology of twisted magnetic configurations in solar flares. Astron. Astrophys. 351, 707-720.

Török, T., Kliem, B., 2005. Confined and ejective eruptions of kink-unstable flux ropes. Astrophys. J. 630, L97-L100.

Török, T., Kliem, B., Titov, V.S., 2004. Ideal kink instability of a magnetic loop equilibrium. Astron. Astrophys. 413, L27-L30.

Tóth, G., Sokolov, I.V., Gombosi, T.I., Chesney, D.R., Clauer, C.R., De Zeeuw, D.L., Hansen, K.C., Kane, K.J., Manchester, W.B., Oehmke, R.C., Powell, K.G., Ridley, A.J., Roussev, I.I., Stout, Q.F., Volberg, O., 2005. Space weather modeling framework: a new tool for the space science community. J. Geophys. Res. 110, A12226.

Tousey, R., 1973. The solar corona. In: Rycroft, M.J., Runcorn, S.K. (Eds.), Space Research, vol. XIII. Akademie-Verlag, Berlin, p. 713.

Usmanov, A.V., Goldstein, M.L., 2006. A three-dimensional MHD solar wind model with pickup protons. J. Geophys. Res. 111, A07101.

van der Holst, B., Jacobs, C., Poedts, S., 2007. Simulation of a breakout coronal mass ejection in the solar wind. Astrophys. J. 671, L77-L80.

van der Holst, B., Keppens, R., 2007. Hybrid block-AMR in cartesian and curvilinear coordinates: MHD applications. J. Comput. Phys. 226, 925-946.

van der Holst, B., Manchester, W.I., Sokolov, I., Toth, G., Gombosi, T.I., DeZeeuw, D., Cohen, O., 2009. Breakout coronal mass ejection or streamer blowout: the bugle effect. Astrophys. J. 693, 1178-1187.
Vandas, M., Odstrćil, D., Watari, S., 2002. Three-dimensional MHD simulation of a loop-like magnetic cloud in the solar wind. J. Geophys. Res. 107, 1236.

Vršnak, B., Ruždjak, D., Sudar, D., Gopalswamy, N., 2004. Kinematics of coronal mass ejections between 2 and 30 solar radii. Astron. Astrophys. 423, 717-728.

Vršnak, B., Žic, T., Falkenberg, T.V., Möstl, C.S.V., Vrbanec, D., 2010. The role of aerodynamic drag in propagation of interplanetary coronal mass ejections. Astron. Astrophys. 512, A43.

Wang, Y.M., Sheeley Jr., N.R., 1990. Solar wind speed and coronal flux-tube expansion. Astrophys. J. 355, 726-732.

Wu, C.C., Fry, C.D., Berdichevsky, D., Dryer, M., Smith, Z., Detman, T., 2005. Predicting the arrival time of shock passages at Earth. Sol. Phys. 227, 371-386.

Wu, C.C., Fry, C.D., Dryer, M., Wu, S.T., Thompson, B., Liou, K., Feng, X.S., 2007a. Threedimensional global simulation of multiple ICMEs' interaction and propagation from the Sun to the heliosphere following the 25-28 October 2003 solar events. Adv. Space. Res. 40, 1827-1834.

Wu, C.C., Fry, C.D., Wu, S.T., Dryer, M., Liou, K., 2007b. Three-dimensional global simulation of interplanetary coronal mass ejection propagation from the Sun to the heliosphere: solar event of 12 May 1997. J. Geophys. Res. 112 9104-+.

Yashiro, S., Gopalswamy, N., Michalek, G., St. Cyr, O.C., Plunkett, S.P., Rich, N.B., Howard, R.A., 2004. A catalog of white light coronal mass ejections observed by the SOHO spacecraft. J. Geophys. Res. 109, A07105.

Yeates, A.R., Mackay, D., 2009. Initiation of coronal mass ejections in a global evolution model. Astrophys. J. 699, 1024-1037.

Zhang, M., Chye Low, B., 2005. The Hydromagnetic Nature of Solar Coronal Mass Ejections. Ann. Rev. Astron. \& Astroph. 43, 103-137.

Zuccarello, F.P., Jacobs, C., Soenen, A., Poedts, S., van der Holst, B., Zuccarello, F., 2009. Modelling the initiation of coronal mass ejections: magnetic flux emergence versus shearing motions. Astron. Astrophys. 507, 441-452.

Zurbuchen, T.H., Richardson, I.G., 2006. In-situ solar wind and magnetic field signatures of interplanetary coronal mass ejections. Space. Sci. Rev. 123, 31-43. 